\title{
Increased Brain Activation for Dual Tasking with 70-Days Head-Down Bed Rest
}

\author{
Peng Yuan ${ }^{1}$, Vincent Koppelmans ${ }^{1}$, Patricia A. Reuter-Lorenz ${ }^{2}$, Yiri E. De Dios ${ }^{3}$, \\ Nichole E. Gadd ${ }^{3}$, Scott J. Wood ${ }^{4}$, Roy Riascos ${ }^{5}$, Igor S. Kofman ${ }^{3}$, Jacob J. Bloomberg ${ }^{6}$, \\ Ajitkumar P. Mulavara ${ }^{6,7}$ and Rachael D. Seidler ${ }^{1,2,8 *}$ \\ ${ }^{1}$ School of Kinesiology, University of Michigan, Ann Arbor, MI, USA, ${ }^{2}$ Department of Psychology, University of Michigan, Ann \\ Arbor, MI, USA, ${ }^{3}$ Wyle Science, Technology and Engineering Group, Houston, TX, USA, ${ }^{4}$ Department of Psychology, Azusa \\ Pacific University, Azusa, CA, USA, ${ }^{5}$ The University of Texas Health Science Center, Houston, TX, USA, ${ }^{6}$ NASA Johnson \\ Space Center, Houston, TX, USA, ${ }^{7}$ Universities Space Research Association, Houston, TX, USA, ${ }^{8}$ Neuroscience Program, \\ Medical School, University of Michigan, Ann Arbor, MI, USA
}

\section{OPEN ACCESS}

Edited by: Mikhail Lebedev,

Duke University, USA

Reviewed by:

Stefano Ramat,

University of Pavia, Italy

Guy Cheron,

Université libre de Bruxelles, Belgium Ana-Maria Cebolla,

Université libre de Bruxelles, Belgium

${ }^{*}$ Correspondence: Rachael D. Seidler rseidler@umich.edu

Received: 01 June 2016 Accepted: 05 August 2016 Published: 23 August 2016

Citation:

Yuan P, Koppelmans $V$ Reuter-Lorenz PA, De Dios YE, Gadd NE, Wood SJ, Riascos R, Kofman IS, Bloomberg JJ, Mulavara AP and Seidler RD (2016) Increased Brain Activation for Dual Tasking with 70-Days Head-Down

Bed Rest.

Front. Syst. Neurosci. 10:71. doi: 10.3389/fnsys.2016.00071
Head-down tilt bed rest (HDBR) has been used as a spaceflight analog to simulate the effects of microgravity exposure on human physiology, sensorimotor function, and cognition on Earth. Previous studies have reported that concurrent performance of motor and cognitive tasks can be impaired during space missions. Understanding the consequences of HDBR for neural control of dual tasking may possibly provide insight into neural efficiency during spaceflight. In the current study, we evaluated how dual task performance and the underlying brain activation changed as a function of HDBR. Eighteen healthy men participated in this study. They remained continuously in the $6^{\circ}$ head-down tilt position for 70 days. Functional MRI for bimanual finger tapping was acquired during both single task and dual task conditions, and repeated at 7 time points pre-, during- and post-HDBR. Another 12 healthy males participated as controls who did not undergo HDBR. A widely distributed network involving the frontal, parietal, cingulate, temporal, and occipital cortices exhibited increased activation for dual tasking and increased activation differences between dual and single task conditions during HDBR relative to pre- or post-HDBR. This HDBR-related brain activation increase for dual tasking implies that more neurocognitive control is needed for dual task execution during HDBR compared to pre- and post-HDBR. We observed a positive correlation between pre-to-post HDBR changes in dual-task cost of reaction time and pre-to-post HDBR change in dual-task cost of brain activation in several cerebral and cerebellar regions. These findings could be predictive of changes in dual task processing during spaceflight.

Keywords: dual task, head-down bed rest, fMRI, microgravity analog, brain activity

\section{INTRODUCTION}

It has been over half a century since the first man traveled in space. Since then, research has revealed a number of physiological and behavioral changes that are associated with spaceflight, such as alterations in muscle, bone, balance, mobility, cardiovascular function, and cognitive performance (Nicogossian et al., 1994; Manzey and Lorenz, 1998; Buckey, 2006; Mulavara et al., 2010; Strangman et al., 2014). Understanding the consequences of space travel could lead to countermeasures to 
mitigate spaceflight-related physical and psychological declines, resulting in improved mission performance.

Several ground-based models have been developed to simulate the spaceflight environment. Each of the analog environments imitates certain specific characteristics of spaceflight. For example, dry immersion is used as an analog of supportlessness (Pavy-Le Traon et al., 2007; Navasiolava et al., 2011); chronically elevated carbon dioxide is employed to simulate elevated $\mathrm{CO}_{2}$ levels in a sealed cabin (Wenzel et al., 1998); and isolated environments such as Antarctic station winter overs are used to mimic the limited social environment of prolonged spaceflight (Lugg, 2005; Basner et al., 2013). Particularly, head-down bed rest (HDBR) has been extensively utilized as an analog to study the effects of cephalic fluid shifts, foot sole unloading, and sensorimotor adaptation that can influence human physiology during exposure to microgravity. As a consequence of being in a head-down tilt supine position for a prolonged period, intravascular and extravascular fluids are shifted to the upper body, and head, as is observed in microgravity (Caprihan et al., 1999; Pavy-Le Traon et al., 2007). HDBR is also associated with foot sole unloading. Studies using the hindlimb unloaded rat model have documented changes in artery morphology and neural activity. For example, hindlimb unloading results in increases in the cross-sectional area and thickness of the basilar artery (Wilkerson et al., 1999), and attenuates baroreflexmediated increases in sympathetic activity (Foley et al., 2005). In addition to fluid shifts and unloading, both spaceflight and HDBR conditions involve a process of adaptation to a new environment, which is associated with sensory reweighting and potentially neuroplastic changes. A series of head-down tilt degrees has been tested, and $-6^{\circ}$ was considered a good balance of acceptability and effect size on physiological changes (At'kov and Bednenko, 1992). Hence, most bed rest studies use headdown tilt of $-6^{\circ}$ to mimic cephalic fluid shifts in addition to axial body unloading.

People often must perform two or more tasks at the same time, e.g., walking while watching traffic lights, talking, eating, etc. Performance in one or both tasks typically declines when two tasks are carried out simultaneously (Strayer et al., 2003), especially when the tasks are not automated. For example, a meta-analysis documented decline of gait performance when a secondary cognitive task was conducted concurrently with walking (Al-Yahya et al., 2011). Such performance declines from single to dual task conditions are typically referred to as dualtask costs. Dual-task cost is thought to reflect the capacity for central processing (Tombu and Jolicoeur, 2003), with lower dualtask costs indicating a larger central capacity, or better cognitive ability. For example, the magnitude of dual-task cost is greater in older adults than in young adults (Verhaeghen et al., 2003; Riby et al., 2004). Dual-task declines also depend on the nature of the task. Motor tasks and tasks requiring substantial control exhibit larger dual-task costs than relatively simpler tasks or tasks that depend on automated processing (Riby et al., 2004; Al-Yahya et al., 2011).

Estimating the influence of spaceflight on dual task performance is important for understanding the constraints on future missions. According to the existing literature, the influence of spaceflight on dual task performance yields mixed results. For example, a single-case study reported impairments in dual task performance of concurrent unstable tracking and memory search during 8 days of spaceflight (Manzey et al., 1995). Another study of simultaneous unstable tracking and a reaction time task reported increased tracking error under dual task conditions during a 5- to 6-month space mission (Bock et al., 2010). In addition to dual task impairments, increased dual-task costs have also been documented. For example, Bock et al. found increased dual-task costs for tracking error during spaceflight (Bock et al., 2010) while a single-subject study also reported increased dual-task cost at the beginning of a space mission compared to pre-flight levels (Manzey et al., 1998). In contrast, a study of a 16-day space mission reported no performance impairments in either simultaneous tracking or manual reaction time performance (Fowler et al., 2000). Studies that have observed increased dual task costs with spaceflight attribute them to stress, fatigue, and sensorimotor adaptation (Manzey and Lorenz, 1998; Fowler et al., 2000; Bock et al., 2010). Although not all studies found a significant influence of spaceflight on dual tasking performance, the effects seen with the increased tracking error and longer reaction time persisted through the duration of spaceflight and even a few days post-spaceflight, raising the importance of effective dual tasking, especially during landing operations.

Dual task studies in spaceflight analog settings are scant. A 45-day HDBR study which examined time-based prospective memory with an ongoing word recall task identified impaired prospective memory during HDBR compared to pre-HDBR (Chen et al., 2013). Nevertheless, no impairment of dual tasking was found during another 17-day HDBR study, which used simultaneous memory search and unstable tracking (Shehab et al., 1998).

The discrepancy of dual task impairments during spaceflight and ground-based analogs could result from the heterogeneity of experiment settings. It is possible that some tasks are more sensitive to space-related changes than other tasks. Furthermore, previous studies had relatively small sample sizes; thus it is also possible that in some studies low statistical power led to null results.

Investigators have used functional neuroimaging to understand the neural basis of dual-task interference. Even when behavioral dual task costs are not evident, additional brain networks may be recruited to meet added demand, resulting in reduced neural efficiency. Although it is suggested that the activated brain regions under dual task conditions depend on the component tasks (Adcock et al., 2000), the prefrontal cortex has been identified as a key region engaged during dual tasking (D’Esposito et al., 1995; Szameitat et al., 2002). Greater activation in the left inferior frontal junction is found for dual task than for single task performance, and this effect decreases with training, implying an association between brain activity and dual tasking ability (Erickson et al., 2007; Dux et al., 2009). In addition to the prefrontal cortex, the parietal cortex, dorsal pre-motor cortex, and anterior cingulate cortex have also been proposed as putative components of a dual task processing network (D'Esposito et al., 1995; Szameitat et al., 2002; Marois et al., 2006). 
Understanding the consequences of HDBR for dual tasking neural activity can provide insight into the influence of spaceflight on cognitive control and neural efficiency, and help to understand the factors affecting adaptation to microgravity. To the best of our knowledge, there are no published studies of the effects of spaceflight or analog environments on dual tasking neural activity. In the current study, subjects simultaneously performed target counting and bimanual button pressing tasks while $\mathrm{fMRI}$ data were acquired before, during and after a 70day HDBR exposure; a protocol paper for this study has been published previously (Koppelmans et al., 2013). We chose to combine a motor and cognitive task because this combination has been investigated previously in spaceflight studies (Manzey et al., 1995; Fowler et al., 2000; Bock et al., 2010). We hypothesized that when compared to the pre-HDBR baseline and in comparison to a control group of subjects not undergoing HDBR: (1) dual task performance would deteriorate during HDBR; and (2) brain activity for dual tasking during HDBR would increase in prefrontal, parietal, pre-motor, and anterior cingulate cortices. We further predicted that activation changes from pre-to-post HDBR in frontal, parietal and cingulate regions would correlate with dual task performance changes.

\section{METHODS}

\section{Participants}

Eighteen healthy male subjects participated in the 70 -day, $6^{\circ}$ HDBR campaign. They were right-handed and aged $31.1 \pm 4.7$ years at the time of admission (range: $25.7-39.8$ years). These subjects were enrolled 13-23 days before the start of HDBR and released 14 days after HDBR. During the 70-days of bed rest, participants remained in the head-down tilt position all the time except for $30 \mathrm{~min}$ at each meal, when they were allowed to support their head with their hand. The study took place in a bed rest facility at the University of Texas Medical Branch, Galveston TX. All subjects received financial compensation for their participation. Thirteen of the HDBR subjects participated in an exercise protocol (Ploutz-Snyder et al., 2014; Koppelmans et al., 2015), which started 20 days before HDBR and continued until the end of HDBR, 6 days per week. The other five HDBR subjects did not exercise. In the current study, all HDBR participants' data were analyzed as one group and exercise was not included as a factor because the majority of HDBR subjects exercised.

Another 12 healthy males participated as control participants not undergoing HDBR who were recruited by the Human Test subject facility at NASA-JSC. These control participants were leading their usual everyday life. These ground-based control subjects were aged $41.4 \pm 9.9$ years at the time of admission (range: 26.2-59.7 years). All the HDBR and control subjects passed an Air Force Class III equivalent physical examination. Both the HDBR study and control study were conducted in accordance with the declaration of Helsinki, and were approved by the institutional review boards of the University of Michigan, the University of Texas-Medical Branch (UTMB), and NASAJSC. Written informed consent was obtained from all the participants.

\section{Cognitive Test}

For HDBR subjects, testing was performed at 7 sessions: $14.1 \pm$ 3.8 days and $7.9 \pm 2.0$ days before the start of HDBR, $8.4 \pm$ 1.0 days, $50.6 \pm 0.9$ days, and $66.8 \pm 1.8$ days after the onset of HDBR, as well as $6.7 \pm 0.8$ days and $11.4 \pm 1.6$ days after HDBR. For control subjects, the task was repeated four times at days $0,12.6 \pm 9.7,50.2 \pm 12.8$, and $84.8 \pm 14.0$. The timelines and deviations are presented in Figure 1.

The finger tapping task was administered under both single and dual task conditions while we acquired fMRI data. In the finger tapping task, two stimulus boxes were presented on the screen. An "x" appeared in one of the two stimulus boxes with an inter-stimulus interval of $800 \mathrm{~ms}$ (Figure 2A). The stimuli were presented randomly, and subjects were instructed to indicate in which one of the stimulus boxes the " $\mathrm{x}$ " appeared by pressing the matching response button with either the right or the left index finger. In a secondary counting task, another stimulus box was presented, which changed color at a rate of $3 \mathrm{~Hz}$ (Figure 2B). Subjects were required to count the number of times that a target color appeared. The incidence of the target color was kept low (1-3\%) so that subjects had to remain vigilant. In some blocks, which were referred to as dual task blocks, subjects were asked to perform the tapping and counting tasks simultaneously (dual task condition, Figure 2C, Seidler et al., 2002). For the dual task condition the box in which the colors were presented was centered and above the two boxes in which the " $\mathrm{x}$ " appeared. In the other blocks, subjects performed the tapping or counting task in isolation (tapping blocks and counting blocks, respectively). Two tapping blocks, two counting blocks, and two dual task blocks were included in an fMRI run, presented in a pseudorandom order. Each block was preceded and followed by 20 -s rest periods, constituting an fMRI run of $260 \mathrm{~s}$. There were two fMRI runs in each session. The latency of tapping timed from the onset of the " $\mathrm{x}$," as well as percent correct of tapping and counting were recorded and analyzed as dependent measures of performance.

\section{Image Acquisition and Processing}

The fMRI scans for HDBR subjects were acquired on a 3Tesla Siemens Magnetom Skyra MRI scanner, and the fMRI for control subjects were acquired on a 3-Tesla Siemens Magnetom Verio MRI scanner. The fMRI scan protocol was identical for all subjects. For the fMRI, a gradient echo T2*-weighted echoplanar imaging (EPI) sequence was used to collect the scans. The following parameters were used: $T R=3660 \mathrm{~ms}, T E=39 \mathrm{~ms}$, flip angle $=90^{\circ}, \mathrm{FOV}=240 \times 240 \mathrm{~mm}$, slice thickness $=4 \mathrm{~mm}$, slice gap $=1 \mathrm{~mm}$, matrix $=94 \times 94$, voxel size $=2.55 \times 2.55$ $\times 5.0 \mathrm{~mm}, 36$ axial slices. A T1-weighted gradient-echo pulse sequence was also collected for all subjects. For HDBR subjects, we used the following parameters: $T R=1900 \mathrm{~ms}, T E=2.49 \mathrm{~ms}$, flip angle $=9^{\circ}, \mathrm{FOV}=270 \times 270 \mathrm{~mm}$, slice thickness $=0.9$ $\mathrm{mm}$; matrix $=288 \times 288$, voxel size $=0.94 \times 0.94 \mathrm{~mm}, 192$ slices; duration $=\sim 4 \mathrm{~min}$. For the control subjects, T1 images were collected with the following parameters: $T R=1900 \mathrm{~ms}$, $\mathrm{TE}=2.32 \mathrm{~ms}$, flip angle $=9^{\circ}, \mathrm{FOV}=250 \times 250 \mathrm{~mm}$, slice thickness $=0.9 \mathrm{~mm}, 192$ slices; matrix $=512 \times 512$, voxel size $=0.49 \times 0.49 \mathrm{~mm}, 3 \mathrm{D} \mathrm{T} 1$ axial overlay; duration $=\sim 4 \mathrm{~min}$. 


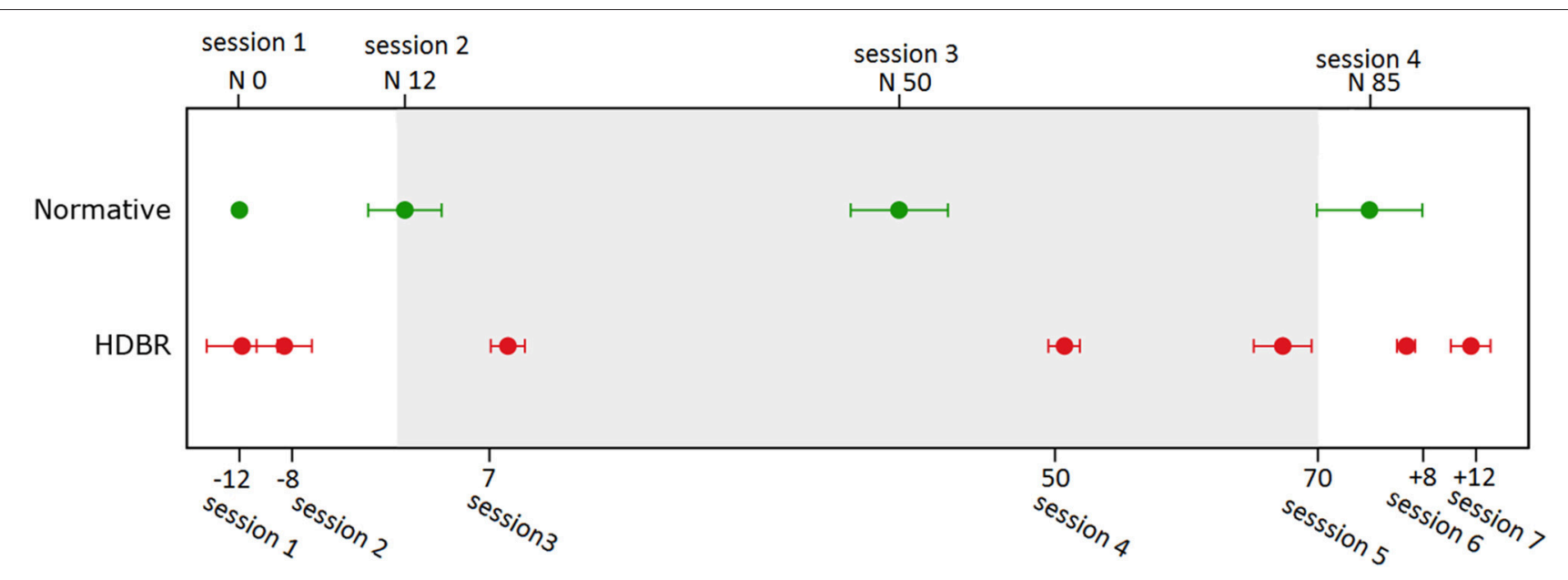

FIGURE 1 | Testing timeline for bed rest subjects and normative control subjects. Error bars represent standard errors.

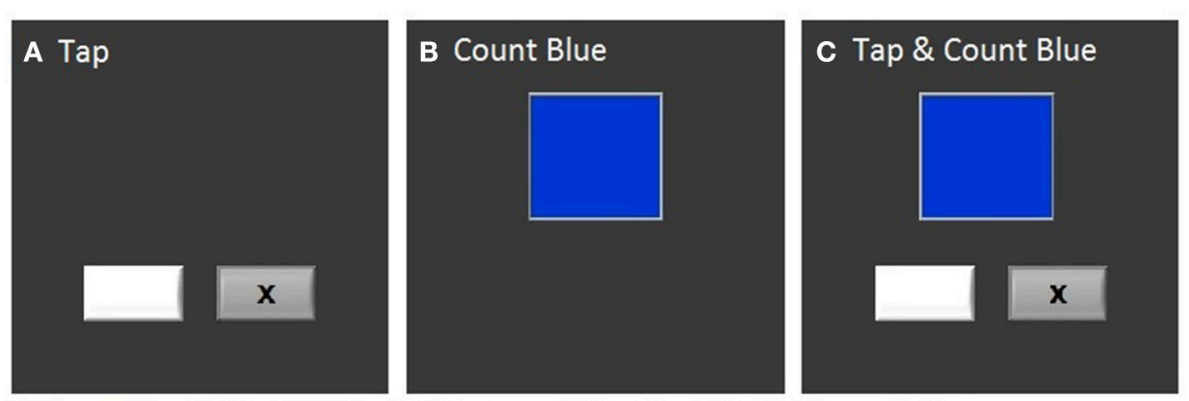

In these examples, you would tap the Right Trigger button.

FIGURE 2 | The dual task test. (A) Finger tapping task, (B) Counting task, (C) Dual task.

The functional images were corrected for slice timing and realigned to correct for head motion. The Artifact Detection Tool (ART, http://www.nitrc.org/projects/artifact_detect/) was used to quantify head motion and detect motion and global brain activation outliers, which were used as nuisance variables in the first level analyses. Then the images were normalized to the Montreal Neurological Institute (MNI) 152 space (Friston et al., 1995) using multiple steps: First, the T1 images were corrected for field inhomogeneity using N4ITK within an intracranial mask obtained from FSL's brain extraction tool (BET; Tustison et al., 2010). Second, the field bias corrected image was skull stripped using FSL's BET, with robust brain center estimation and a fractional intensity threshold of 0.1 . Third, the skull stripped T1 images were co-registered to the mean fMRI EPI using SPM8. Fourth, advanced normalization tools (ANTs) were used to normalize the co-registered images to MNI152 common space (Avants et al., 2011). Then the warping parameters were applied for spatial smoothing with an 8 -mm full-width halfmaximum 3-dimensional Gaussian kernel. In addition to the whole brain normalization, a spatially unbiased atlas template of the cerebellum and brainstem (SUIT; Diedrichsen, 2006) was used for cerebellar normalization. fMRI runs with large head motion ( $>3 \mathrm{~mm}$ ) were omitted.
The functional data were analyzed using SPM8. In the first level analysis, we calculated brain activity for each participant on a voxel-by-voxel basis for: tapping under the single task condition versus rest, the dual task condition versus rest, as well as dual task cost of brain activation, which is the additional brain activity under dual tasking above and beyond that observed in the single task condition.

In the second level analyses, flexible factorial (SPM's mixed model equivalent) analysis was used to determine brain activity changes across sessions. We set several contrast vectors to test the hypothesized relative level of brain activation in each session. For the HDBR subjects, we sought to identify brain areas with two types of HDBR-related activation changes: immediate change and cumulative change. The immediate change was assumed to be sensitive to HDBR status, and was hypothesized to take place shortly after the start of HDBR, to be maintained during HDBR, and to restore shortly after the finish of HDBR (Figure 3A). The cumulative change was presumed to increase progressively with the days in HDBR, peaking at the end of HDBR, and recovering gradually after HDBR (Figure 3B). The first measurement session was regarded as a practice session, thus the fMRI data in the first session was excluded. For HDBR subjects, the activation levels in the later six sessions (2nd to 7th) 

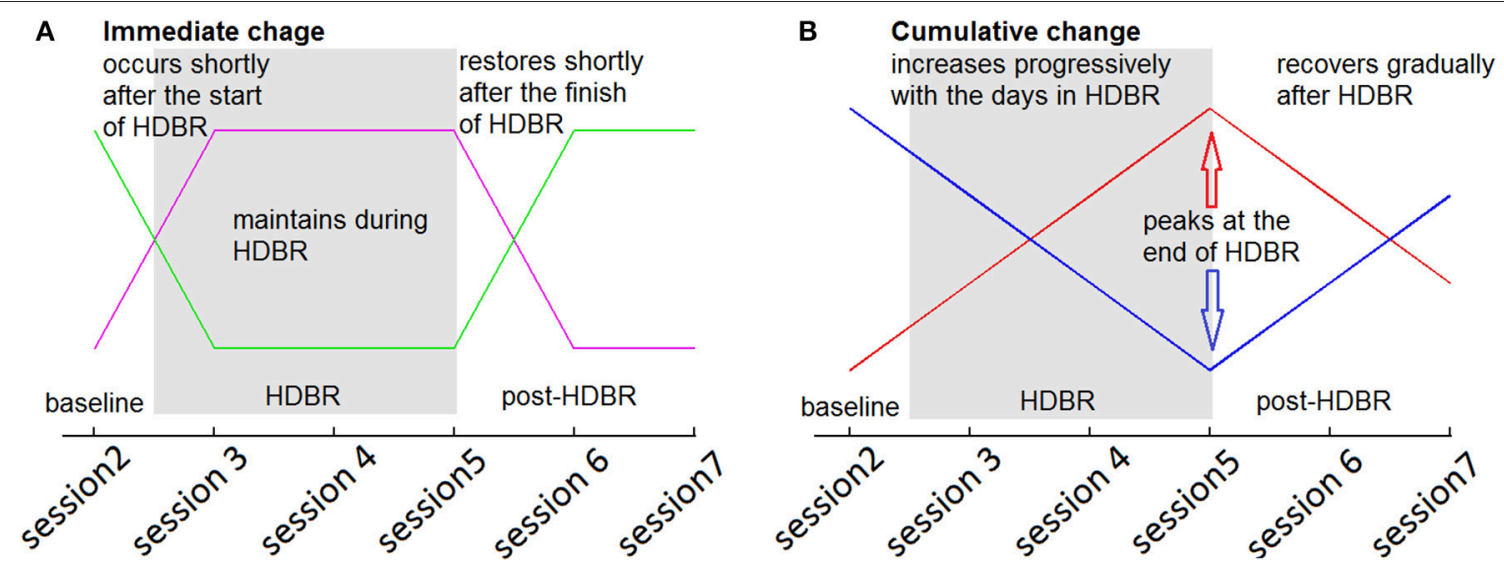

FIGURE 3 | Hypothesized HDBR-related brain activation changes. (A) Immediate increase (violet) and immediate decrease (green). (B) Cumulative increase (red) and cumulative decrease (blue).

were evaluated using contrast vectors as weights. For the control subjects, the first session was also regarded as practice. Therefore, we estimated linear increases and decreases in brain activation across the 2nd, 3rd, and 4th sessions for control subjects to evaluate any further practice effects.

In the second level analyses for HDBR subjects, we also calculated the brain activation difference between the baseline (2nd session, $7.9 \pm 2.0$ days before HDBR) and the end of HDBR (5th session, $66.8 \pm 1.8$ days in HDBR), in order to identify brain regions in which the activation change was significantly associated with behavioral change. For all fMRI analyses, the alpha level was set at 0.001 (uncorrected for multiple comparisons), and the extent threshold was 10 voxels.

\section{Statistical Analyses for Behavioral Data}

Linear mixed model analysis was used to compare the time courses of behavioral change in HDBR versus control subjects. Subject number was entered as a random variable. Time was entered as the number of days from the second session, which was treated as a continuous variable. The potential age effect on behavior was controlled statistically. For HDBR subjects, data from the post-HDBR sessions were excluded so we could examine whether the slope of change within bed rest differed from any potential practice effects in the control subjects. For all subjects, the data in the first session was dropped as a practice. Thus the behavioral data in the 2nd, 3rd, 4th, and 5th sessions (baseline and during HDBR) for HDBR subjects and behavioral scores in the 2nd, 3rd, and 4th sessions for control subjects were included. Restricted maximum likelihood (REML) was used. We used Stata 14 to analyze the behavioral data, and set the alpha level at 0.05 . Bonferroni correction was used to correct for multiple comparisons.

\section{RESULTS}

\section{Behavioral Results}

There was no effect of age on any of the behavioral scores, so it was not considered in any additional analyses. HDBR

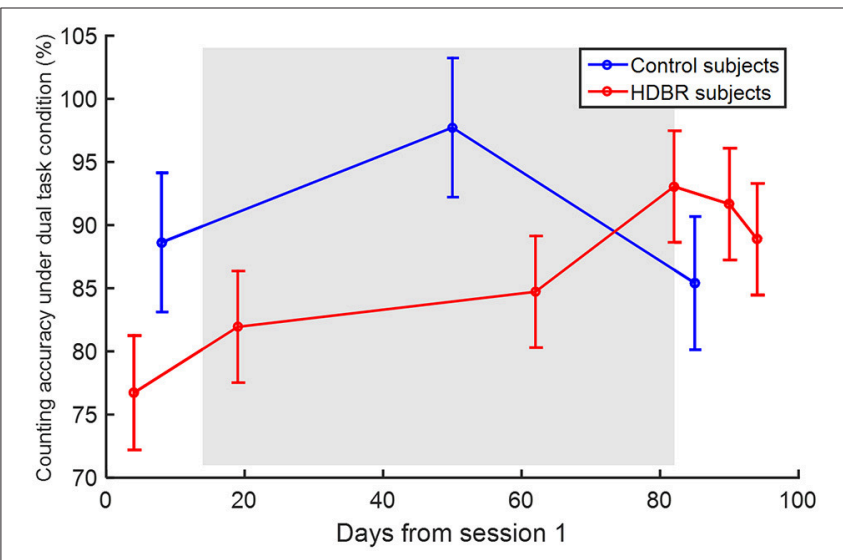

FIGURE 4 | Counting accuracy under the dual task condition. Error bars represent the standard errors.

subjects showed a lower counting accuracy under the dual task condition $(\beta=-17.807, p=0.038)$ than control subjects. There was also a significant interaction of group $\times$ time $(\beta=0.315$, $p=0.048)$, indicating that the HDBR subjects showed greater improvement in counting accuracy under the dual task condition than the control subjects. The counting accuracies are illustrated in Figure 4.

\section{fMRI Results}

\section{Brain Activation for Dual Task at Baseline}

The average HDBR group brain activation and deactivation for dual tasking at session 2 is shown in Figure 5. Consistent with the literature, we observed a distributed network involving the frontal, parietal, occipital, temporal, and cerebellar regions activated while subjects performed the dual task.

\section{Brain Activation Change for Tapping under Single Task Condition}

A few brain areas exhibited significant HDBR-related cumulative increases or decreases in activation for tapping under the single 
task condition (Figure 6, Table 1). However, no regions exhibited significant immediate change.

For the control subjects, several brain regions exhibited increases or decreases in activation for tapping under the single task condition from the 2 nd session to the 4 th session (Table 2), but nevertheless these regions did not overlap with those showing HDBR-related cumulative changes.

\section{Brain Activation Change for Tapping under Dual Task Condition}

For tapping under the dual task condition, a few regions in the parietal lobe and thalamus exhibited HDBR-related cumulative increases in activation (Figure 7, Table 3). The brain regions with immediate HDBR-related increases were distributed in the frontal, parietal, occipital, and temporal lobes, as well as cerebellum and subcortical regions (Figure 7, Table 4). However, no HDBR-related immediate decrease or cumulative decrease was found. There was no significant activation difference between the baseline (2nd session) and the last post-HDBR session (7th session) in the brain areas showing HDBR-associated activity change.

In contrast, in the control subjects several regions in frontal cortex, occipital cortex, cerebellum, and putamen showed decreased activation for dual tasking from session 2 to session 4 (Table 5), however no activation increase from session 2 to session 4 was found. The opposing direction of these changes versus those seen in the HDBR subjects supports the specificity of HDBR effects resulting from the intervention itself rather than practice effects.

\section{Brain-Behavioral Correlation for Tapping under Dual Task Condition}

The pre-to-post HDBR difference in tapping reaction time (dual task condition) was positively associated with pre-to-post HDBR

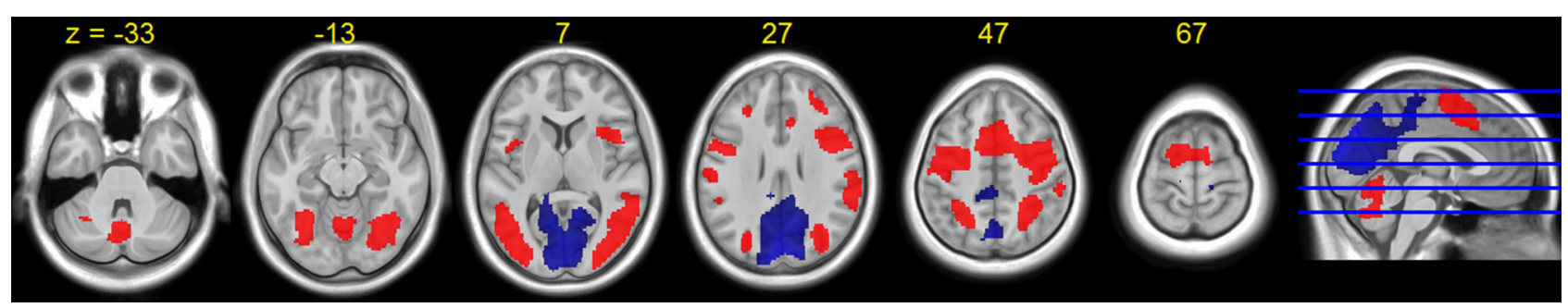

FIGURE 5 | HDBR group T-statistic maps for brain activation (red) and deactivation (blue) for dual task at baseline (session 2). Thresholded at abs ( 7 ) > 3.218 .

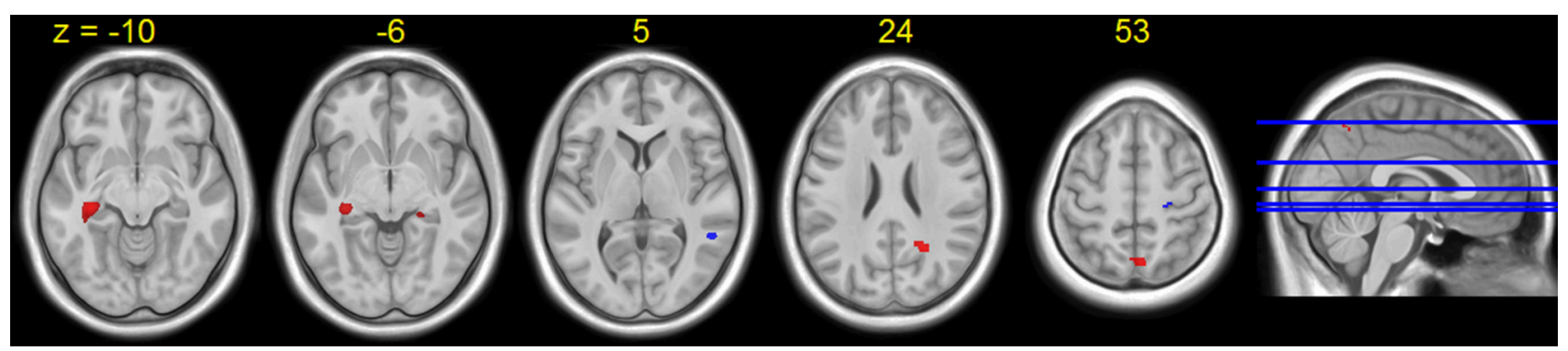

FIGURE 6 | Regions showing HDBR-related cumulative activation change for tapping under the single task condition. Red: HDBR-related increase; blue: HDBR-related decrease. Group T-statistic map, thresholded at abs $(T)>3.218$.

TABLE 1 | Regions showing HDBR-related cumulative activation changes for tapping under the single task condition.

\begin{tabular}{|c|c|c|c|c|c|c|c|c|}
\hline & Region & & $k$ & Peak $T$ & Peak $p$ & & , z (mm & \\
\hline \multirow[t]{4}{*}{ Increase } & Parietal & R Precuneus & 36 & 3.732 & $2 \mathrm{E}-04$ & 24 & -56 & 24 \\
\hline & & R Precuneus & 55 & 3.609 & 3E-04 & 6 & -68 & 52 \\
\hline & Temporal & L Hippocampus & 996 & 5.090 & $1 \mathrm{E}-06$ & -33 & -26 & -10 \\
\hline & & R Parahippocampal Gyrus & 69 & 3.432 & $5 E-04$ & 22 & -32 & -6 \\
\hline \multirow[t]{2}{*}{ Decrease } & Frontal & R Precentral Gyrus & 10 & 3.380 & 5E-04 & 22 & -26 & 54 \\
\hline & Temporal & R Middle Temporal Gyrus & 125 & 3.531 & 3E-04 & 51 & -47 & 5 \\
\hline
\end{tabular}

k, cluster size; peak T, t-value at the peak voxel; peak p, p-value at the peak voxel; $x, y, z$, MNI coordinates of the peak voxel. 
TABLE 2 | Regions showing activation changes for tapping under the single task condition in control subjects from sessions 2 to 4.

\begin{tabular}{|c|c|c|c|c|c|c|c|c|}
\hline & Region & & $k$ & Peak $T$ & Peak $p$ & & $z(\mathrm{~mm}$ & \\
\hline \multirow[t]{3}{*}{ Increase } & Parietal & L precuneus & 479 & 5.095 & 6E-06 & -12 & -54 & 12 \\
\hline & Cingulate & L Middle Cingulate Gyrus & 31 & 3.784 & 3E-04 & -4 & -32 & 38 \\
\hline & Cerebellum & L Cerebellum_Lobule VIII & 56 & 3.568 & 5E-04 & -25 & -51 & -39 \\
\hline \multirow[t]{2}{*}{ Decrease } & Frontal & R Superiour Frontal Gyrus & 10 & 3.609 & 5E-04 & 16 & 6 & 54 \\
\hline & Temporal & R Hippocampus & 114 & 4.022 & 2E-04 & 37 & -25 & -6 \\
\hline
\end{tabular}

k, cluster size; peak T, t-value at the peak voxel; peak p, p-value at the peak voxel; $x, y, z$, MNI coordinates of the peak voxel.
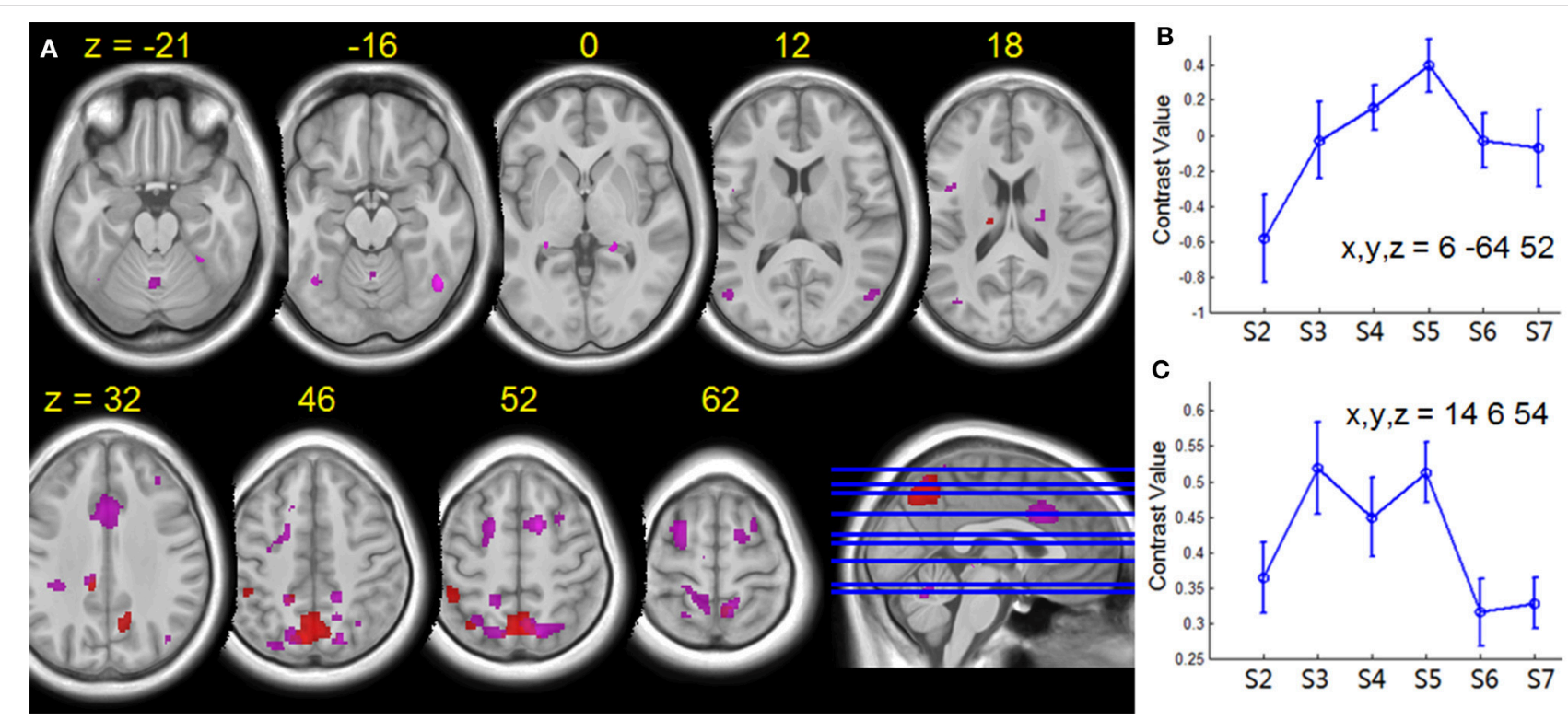

FIGURE 7 | (A) Group T-statistic maps for the regions showing HDBR-related changes in activation for dual tasking, thresholded at abs (T) > 3.218. Red: HDBR-related cumulative increases; violet: HDBR-related immediate increases. (B) Example of cumulative increase across sessions. (C) Example of immediate increase across sessions. The MNI coordinates of the sample regions are indicated in (B,C). Error bars represent standard errors across all the HDBR subjects.

changes of brain activation in a few clusters in the frontal, parietal, temporal, and occipital lobes (Table 6), i.e., greater slowing of reaction time was associated with greater activation increases in these regions. The opposite relationship was found in three clusters within the brain stem and cerebellum (Table 6).

\section{Brain Activation Change for Dual-Task Cost on Tapping}

HDBR-related cumulative increases in dual-task cost of brain activation was observed in frontal, parietal, temporal, and cerebellar areas (Figure 8, Table 7). A cluster in the left hippocampus showed cumulative decreases during HDBR.

In contrast to the cumulative change regions, regions with HDBR-related immediate increase in dual-task cost exhibited a more frontal distribution. In addition to parietal, occipital, and temporal activation, several clusters were observed in frontal and cingulate cortices (Figure 8, Table 8). No immediate decrease was observed during HDBR.

For the control group, no increase in the dual-task cost for brain activation was found from session 2 to session 4 . One cluster within the left cerebellum (crus I, 98 voxels) exhibited decreased dual-task cost of brain activation from session 2 to session 4 (peak $T=3.792$ ). This cluster did not overlap with the brain areas that showed HDBR-related change in dual-task cost of brain activation.

\section{Brain-Behavioral Correlation for Dual-Task Cost of Tapping}

We observed a positive correlation between pre-to-post HDBR changes in dual-task cost on tapping reaction time and dualtask cost on brain activation in a distributed network, involving frontal, parietal, occipital, temporal, insula, and cerebellum areas (Table 9).

\section{DISCUSSION}

The current study investigated the effect of long-term HDBR on dual task performance and the underlying brain activation. The frontal, parietal, cingulate, and temporal cortices exhibited increased activation for dual task execution during HDBR, which 
TABLE 3 | Regions showing HDBR-related cumulative increases in activation for dual tasking.

\begin{tabular}{llcccccc}
\hline Region & & $\boldsymbol{k}$ & Peak $\boldsymbol{T}$ & Peak $\boldsymbol{p}$ & \multicolumn{2}{c}{$\boldsymbol{x}, \boldsymbol{y}, \boldsymbol{z}(\mathbf{m m})$} \\
\hline Parietal & R Precuneus & 1042 & 4.834 & $3 \mathrm{E}-06$ & 6 & -64 & 52 \\
& L Inferior & 86 & 3.846 & $1 \mathrm{E}-04$ & -44 & -42 & 50 \\
& Parietal Lobule & & & & & & \\
& L Precuneus & 33 & 3.669 & $2 \mathrm{E}-04$ & -16 & -46 & 46 \\
& $\begin{array}{l}\text { L Superior } \\
\text { Parietal Lobule }\end{array}$ & 28 & 3.626 & $2 \mathrm{E}-04$ & -32 & -64 & 54 \\
& L Precuneus & 10 & 3.287 & $7 \mathrm{E}-04$ & -30 & -76 & 42 \\
\hline Cingulate & L Posterior & 36 & 3.876 & $1 \mathrm{E}-04$ & -10 & -34 & 32 \\
& Cingulate Gyrus & & & & & & \\
\hline Thalamus & L Thalamus & 10 & 3.588 & $3 \mathrm{E}-04$ & -12 & -18 & 18 \\
\hline
\end{tabular}

k, cluster size; peak T, $t$-value at the peak voxel; peak p, p-value at the peak voxel; $x, y, z$, MNI coordinates of the peak voxel.

then recovered post HDBR, suggesting reduced neural efficiency in a spaceflight analog environment.

We observed activation in the frontal, parietal, and cingulate cortices during dual tasking at the pre-HDBR baseline test, consistent with the existing literature (D'Esposito et al., 1995; Szameitat et al., 2002; Marois et al., 2006). Immediate and cumulative HDBR-related increases of brain activation for dual tasking revealed that more neural resources were required for dual tasking during HDBR. These findings suggest that HDBR may result in lower efficiency of neural processing, which then recovered after HDBR. Bock attributes increased dual task costs inflight to the resource demands of adapting to microgravity (Bock et al., 2010); adaptation to the bed rest environment here may similarly limit resource availability. This change could result from physiological, perceptual, and psychological effects of bed rest. First, when a subject is in a head-down tilt supine position for a sustained period, the intravascular and extravascular fluids are shifted toward the head and upper body. Reports of changes in gray matter volume with HDBR suggest that fluid shifts and the brain settles into a new position toward the back of the skull (Roberts et al., 2010; Rao et al., 2014; Koppelmans et al., under review). The axial body unloading and potential sensory reweighing that occur during $\mathrm{HDBR}$ could tax the available resources. Both HDBR and spaceflight environments challenge the brain to adaptively change and this adaptive alteration may impact dual tasking performance. Second, participants exhibit changes in sensorimotor performance from pre to post HDBR which suggest sensory reweighting and adaptive neuroplasticity (Koppelmans et al., 2015). The resource demands of this process may limit dual tasking abilities. Third, the stress of being in an altered and somewhat isolated environment may also influence subjects' performance and corresponding brain activation patterns. Future studies could directly measure fluid shifts or changing cortisol levels with HDBR to determine how these relate to the increased neural dual task costs we observed here.

The HDBR-associated lower neural efficiency for dual tasking parallels findings from electroencephalographic (EEG) spaceflight studies. Cheron et al. recorded EEG activity during visual processing, and reported decreased event-related synchronization in the theta-alpha oscillations during spaceflight (Cheron et al., 2014). As the EEG alpha component amplitude is negatively associated with BOLD signals (Feige et al., 2005), the reduced alpha power for visual processing would be expected to link with increased BOLD activation (Cheron et al., 2014), and decreased neural efficiency during spaceflight. Cheron et al. also measured spontaneous EEG (while participants were not performing a task) and found that the alpha power increased in the eyes-closed state but did not significantly change in the eyesopen state during spaceflight (Cheron et al., 2006), ruling out the existence of general reductions of alpha power in microgravity. The reduction of neural efficiency for dual tasking in our current study thus supports Cheron's previous findings that neural efficiency for task processing decreases during spaceflight.

When comparing dual task performance of HDBR subjects during HDBR with the performance of control subjects over a similar time course, we observed lower accuracy of counting in the HDBR group than in the control group, but no group difference in the other cognitive scores such as tapping latency and accuracy. The relative retention of dual tasking performance reflects greater frontal and parietal recruitment to maintain the same performance level during HDBR.

We found that dual task processing is more susceptible to HDBR than single task performance: Behaviorally, the HDBRassociated group difference (counting accuracy) was only evident under the dual task condition rather than the single task one. Moreover, the immediate HDBR-related brain activation increase was found for dual tasking but not for single tasking. This is similar to what has been observed in terms of behavior inflight; manual sensorimotor impairments are more evident under dual tasking conditions (Manzey et al., 1995, 1998; Bock et al., 2010). Thus our findings suggest that the spaceflightinduced declines in dual tasking could at least partly be attributed to cephalic fluid shifts, axial body unloading, and sensorimotor adaptation, which were at least partially simulated by HDBR. Spaceflight-specific factors other than those mentioned might of course also contribute to spaceflight-induced declines in dual tasking.

The effect of HDBR on brain activation takes place very quickly, even within 7 days after the onset of HDBR, as expressed by the immediate HDBR-related change in activation for dual tasking. As illustrated in Figure 7, the immediate HDBR-related activation increase for dual tasking was mainly distributed in the frontal, parietal, and cingulate regions-the brain areas that are important for dual task processing. On the other hand, the cumulative HDBR-related activation change for dual tasking was focused at the parietal area especially in the precuneus. In referring to the baseline deactivation regions (Figure 5), the cumulative HDBR-related activation change in Figure 7 is more likely to be due to HDBR-related decreases in deactivation, rather than increases in activation. It has been reported that the precuneus area exhibits gray matter volumetric increases with HDBR (Koppelmans et al., under review), thus it is possible that HDBR-related changes in functional activation could partly be due to HDBR-related 
TABLE 4 | Regions showing HDBR-related immediate increases in activation for dual tasking.

\begin{tabular}{|c|c|c|c|c|c|c|c|}
\hline \multirow{2}{*}{$\begin{array}{l}\text { Region } \\
\text { Frontal }\end{array}$} & \multirow[b]{2}{*}{ R Supplementary Motor Cortex } & \multirow{2}{*}{$\begin{array}{c}\boldsymbol{k} \\
369\end{array}$} & \multirow{2}{*}{$\begin{array}{c}\text { Peak T } \\
5.539\end{array}$} & \multirow{2}{*}{$\begin{array}{l}\text { Peak } \boldsymbol{p} \\
\text { 2E-07 }\end{array}$} & \multicolumn{3}{|c|}{$x, y, z(\mathrm{~mm})$} \\
\hline & & & & & 14 & 6 & 54 \\
\hline & L Superior Frontal Gyrus & 378 & 4.466 & 1E-05 & -24 & 4 & 62 \\
\hline & L Operculum & 24 & 3.629 & $2 \mathrm{E}-04$ & -44 & 2 & 16 \\
\hline & R Middle Frontal Gyrus & 43 & 3.611 & 3E-04 & 36 & 36 & 34 \\
\hline & L Superior Frontal Gyrus & 21 & 3.445 & 4E-04 & -16 & 14 & 40 \\
\hline & L Supplementary Motor Cortex & 10 & 3.428 & $5 E-04$ & -8 & -16 & 58 \\
\hline \multirow[t]{4}{*}{ Parietal } & R Precuneus & 987 & 4.393 & 2E-05 & 14 & -68 & 52 \\
\hline & R Precuneus & 57 & 4.174 & 4E-05 & 16 & -44 & 46 \\
\hline & L Inferior Parietal White Matter & 59 & 3.711 & 2E-04 & -32 & -36 & 32 \\
\hline & R Precuneus & 17 & 3.469 & 4E-04 & 16 & -56 & 46 \\
\hline \multirow[t]{2}{*}{ Cingulate } & R Anterior Cingulate Gyrus & 405 & 4.299 & 2E-05 & 2 & 16 & 30 \\
\hline & L Posterior Cingulate Gyrus & 24 & 3.879 & $1 E-04$ & -12 & -32 & 32 \\
\hline \multirow[t]{4}{*}{ Occipital } & L Middle Occipital Gyrus & 11 & 3.521 & 3E-04 & -20 & -88 & 4 \\
\hline & L Middle Occipital Gyrus & 28 & 3.420 & 5E-04 & -34 & -74 & 22 \\
\hline & R Middle Occipital Gyrus & 11 & 3.306 & 7E-04 & 40 & -72 & 32 \\
\hline & L Lingual Gyrus & 12 & 3.369 & 6E-04 & -3 & -90 & -17 \\
\hline \multirow[t]{6}{*}{ Temporal } & L Middle Temporal Gyrus & 27 & 3.724 & 2E-04 & -48 & -68 & 12 \\
\hline & R Middle Temporal Gyrus & 71 & 3.566 & 3E-04 & 48 & -70 & 12 \\
\hline & L Fusiform Gyrus & 137 & 3.437 & 4E-04 & -38 & -59 & -16 \\
\hline & R Fusiform Gyrus & 469 & 4.154 & 4E-05 & 46 & -59 & -16 \\
\hline & R Parahippocampal Gyrus & 183 & 3.910 & 9E-05 & 20 & -36 & -1 \\
\hline & L Hippocampus & 36 & 3.385 & $5 E-04$ & -26 & -35 & 0 \\
\hline \multirow[t]{2}{*}{ Subcortical } & L Pulvinar Thalamus & 53 & 3.570 & 3E-04 & -4 & -30 & -3 \\
\hline & R Caudate & 18 & 3.823 & 1E-04 & 20 & -14 & 20 \\
\hline \multirow[t]{3}{*}{ Cerebellum } & R Cerebellum Lobule VI & 94 & 3.594 & 3E-04 & 31 & -44 & -21 \\
\hline & Vermis VI & 55 & 3.362 & 6E-04 & -1 & -63 & -22 \\
\hline & Vermis VII & 21 & 3.248 & 8E-04 & 3 & -78 & -27 \\
\hline
\end{tabular}

k, cluster size; peak T, t-value at the peak voxel; peak p, p-value at the peak voxel; $x, y, z$, MNI coordinates of the peak voxel.

TABLE 5 | Regions showing decreases from session 2 to session 4 in activation for dual tasking in the control subjects.

\begin{tabular}{|c|c|c|c|c|c|c|c|}
\hline Region & & $k$ & Peak $T$ & Peak $p$ & & $\mathrm{y}, \mathrm{z}(\mathrm{mn}$ & \\
\hline \multirow[t]{3}{*}{ Frontal } & R Superior Frontal Gyrus & 30 & 4.173 & 1E-04 & 22 & 0 & 52 \\
\hline & L Supplementary Motor Cortex & 31 & 4.064 & 1E-04 & -4 & 8 & 42 \\
\hline & R Supplementary Motor Cortex & 14 & 3.667 & 4E-04 & 12 & 4 & 50 \\
\hline \multirow[t]{5}{*}{ Occipital } & L Inferior Occipital Gyrus & 25 & 4.148 & 1E-04 & -42 & -84 & -8 \\
\hline & R Fusiform Gyrus & 24 & 3.979 & 2E-04 & 42 & -66 & -16 \\
\hline & L Fusiform Gyrus & 208 & 3.842 & 3E-04 & -46 & -59 & -20 \\
\hline & L Middle Occipital Gyrus & 62 & 3.717 & 4E-04 & -30 & -78 & 22 \\
\hline & R Middle Occipital Gyrus & 37 & 3.555 & 6E-04 & 30 & -76 & 28 \\
\hline Subcortical & L Putamen & 10 & 3.712 & 4E-04 & -22 & 22 & -4 \\
\hline Cerebellum & L Cerebellum Lobule VI & 393 & 4.433 & 5E-05 & -33 & -74 & -19 \\
\hline
\end{tabular}

k, cluster size; peak T, $t$-value at the peak voxel; peak p, p-value at the peak voxel; $x, y, z$, MNI coordinates of the peak voxel. 
TABLE 6 | Regions showing associations between pre-to-post HDBR differences in tapping reaction time and brain activation for dual tasking.

\begin{tabular}{|c|c|c|c|c|c|c|c|c|}
\hline & Region & & $\boldsymbol{k}$ & Peak T & Peak $p$ & & $y, z(m n$ & \\
\hline \multirow[t]{5}{*}{ Positive correlation } & Frontal & R Middle Frontal Gyrus & 44 & 5.386 & 8E-05 & 16 & 36 & 32 \\
\hline & & R Middle Frontal Gyrus & 28 & 4.585 & 3E-04 & 40 & 12 & 38 \\
\hline & Parietal & R Precuneus & 82 & 5.373 & 8E-05 & 10 & -54 & 38 \\
\hline & Temporal & L Superior Temporal Gyrus & 24 & 4.869 & 2E-04 & -58 & -14 & -2 \\
\hline & Occipital & R Inferior Occipital Gyrus & 43 & 4.396 & 4E-04 & 19 & -93 & -8 \\
\hline \multirow[t]{3}{*}{ Negative correlation } & Brain stem & Nuclei Gracile & 248 & 5.089 & 1E-04 & 0 & -41 & -58 \\
\hline & & Cuneate Nuclei & 28 & 4.390 & 4E-04 & 4 & -50 & -60 \\
\hline & Cerebellum & Cerebellar Tonsil & 17 & 4.317 & 5E-04 & -7 & -34 & -43 \\
\hline
\end{tabular}

k, cluster size; peak T, $t$-value at the peak voxel; peak p, p-value at the peak voxel; $x, y, z$, MNI coordinates of the peak voxel.

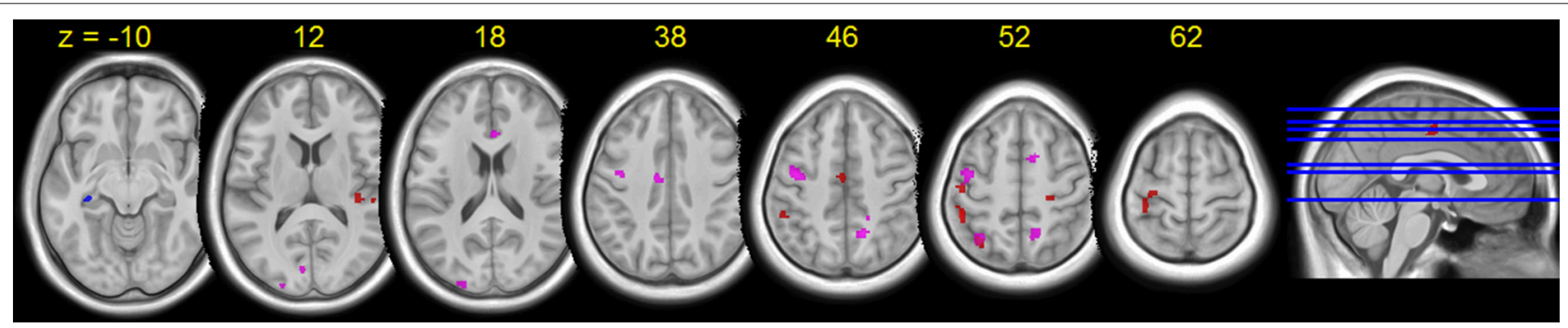

FIGURE 8 | Group T-statistic maps for the regions showing HDBR-related changes in dual tasking cost of brain activation, thresholded at abs (T) > 3.218. Red: HDBR-related cumulative increases; blue: HDBR-related cumulative decreases; violet: HDBR-related immediate increases.

alterations in cortical structure. Our results of the immediate HDBR-associated activation change revealed that the HDBR effects occurred as soon as 7 days in HDBR, however, determining the onset of neural activation change would require further investigation with more frequent measurements. The temporal dynamics of HDBR-related behavioral and neural changes would be more precisely quantified if the behavioral and fMRI measures were collected more frequently, e.g., daily.

The brain regions showing activation changes from session 2 to session 4 in the control subjects did not overlap with the regions showing HDBR-related changes, implying that the latter were not merely due to practice effects. Moreover, the brain activations for dual task and dualtask cost prevailingly increased in certain areas with HDBR, whereas in control participants brain activation decreased with repeated measurements. The opposing directions of these changes support the specificity of the HDBR effects and their mechanism as due to the HDBR intervention but not practice. Furthermore, according to previous reports (Erickson et al., 2007; Wu et al., 2013), if practice did play a role in the functional activation change, then such a practice-related change should be reductions in activation. The observed activation reduction in control subjects likely reflects practice-induced improvements in neural efficiency, which is consistent with previous literature.
The training-induced reduction in brain activation may represent improvement in the efficiency of dual task processing. Previous studies on dual task training have reported an association between brain activation decreases and performance improvements for dual tasking (Erickson et al., 2007; Wu et al., 2013). Thus, the reduction in brain activation could indicate increased neural efficiency, i.e., less neural resources are needed to complete the task (Wu et al., 2013). In light of this literature, subjects in the current study who obtained greater behavioral improvement from repeated training would have a tendency to exhibit greater enhancement in neural efficiency, and then show more reduction in brain activation. Such an inference was supported by our results. We observed a positive correlation between pre-to-post HDBR change in dual-task cost for tapping reaction time and pre-to-post HDBR activation change in frontal, parietal, temporal, and cingulate areas, which verified our hypothesis. This brain-behavior association could be used to predict behavioral change from pre-to-post HDBR changes in the activation of certain brain areas, i.e., subjects with more pre-topost HDBR increase in brain activation are likely to exhibit larger increase in dual-task cost for tapping reaction time.

The current study investigated the effects of HDBR on brain activation for dual tasking, and provided insight into the effects of spaceflight on neural efficiency. However, generalizing the HDBR findings to spaceflight should be approached with caution. First, as an analog environment, HDBR only mimics particular 
TABLE 7 | Regions showing HDBR-related cumulative increases in dual-task costs of brain activation.

\begin{tabular}{|c|c|c|c|c|c|c|c|}
\hline Region & & $\boldsymbol{k}$ & Peak $T$ & Peak $p$ & & $\mathrm{r}, \mathrm{z}(\mathrm{mn}$ & \\
\hline \multirow[t]{4}{*}{ Parietal } & L Inferior Parietal Gyrus & 87 & 4.760 & 4E-06 & -50 & -40 & 50 \\
\hline & L Postcentral Gyrus & 87 & 3.792 & 1E-04 & -36 & -34 & 62 \\
\hline & L Superior Parietal Gyrus & 41 & 3.722 & 2E-04 & -32 & -62 & 52 \\
\hline & L Postcentral Gyrus & 21 & 3.654 & $2 \mathrm{E}-04$ & -48 & -20 & 52 \\
\hline Frontal & R Precentral Gyrus & 24 & 3.565 & 3E-04 & 24 & -26 & 54 \\
\hline Cingulate & L Middle Cingulate Gyrus & 47 & 3.507 & 4E-04 & -4 & -10 & 44 \\
\hline \multirow[t]{2}{*}{ Temporal } & R Middle Temporal Gyrus & 176 & 3.540 & 3E-04 & 49 & -44 & 0 \\
\hline & R Superior Temporal Gyrus & 10 & 3.323 & 7E-04 & 50 & -30 & 10 \\
\hline Insula & R Insula & 22 & 3.497 & 4E-04 & 38 & -26 & 14 \\
\hline Cerebellum & R Cerebellum Lobules IV \& V & 11 & 3.226 & 9E-04 & 19 & -45 & -27 \\
\hline
\end{tabular}

k, cluster size; peak T, t-value at the peak voxel; peak p, p-value at the peak voxel; $x, y, z$, MNI coordinates of the peak voxel.

TABLE 8 | Regions showing HDBR-related immediate increases in dual-task costs of brain activation.

\begin{tabular}{|c|c|c|c|c|c|c|c|}
\hline Region & & $k$ & Peak $T$ & Peak $p$ & & $z(\mathrm{~mm}$ & \\
\hline \multirow[t]{5}{*}{ Frontal } & L Precentral Gyrus & 192 & 3.984 & 7E-05 & -40 & -8 & 46 \\
\hline & R Supplementary Motor Cortex & 41 & 3.963 & 8E-05 & 12 & 4 & 54 \\
\hline & R Middle Frontal Gyrus & 17 & 3.509 & $4 \mathrm{E}-04$ & 26 & -6 & 42 \\
\hline & L Middle Frontal Gyrus & 17 & 3.426 & $5 E-04$ & -44 & 16 & 24 \\
\hline & L Precentral Gyrus & 15 & 3.364 & $6 \mathrm{E}-04$ & -42 & 4 & 32 \\
\hline \multirow[t]{3}{*}{ Parietal } & R Precuneus & 106 & 3.963 & $8 \mathrm{E}-05$ & 14 & -56 & 46 \\
\hline & L Superior Parietal Lobule & 73 & 3.832 & $1 \mathrm{E}-04$ & -32 & -60 & 52 \\
\hline & R Precuneus & 10 & 3.486 & $4 \mathrm{E}-04$ & 20 & -44 & 44 \\
\hline \multirow[t]{3}{*}{ Cingulate } & R Anterior Cingulate Gyrus & 35 & 3.842 & $1 \mathrm{E}-04$ & 4 & 24 & 20 \\
\hline & L Middle Cingulate Gyrus & 34 & 3.773 & $1 \mathrm{E}-04$ & -10 & -12 & 38 \\
\hline & R Middle Cingulate Gyrus & 20 & 3.529 & 3E-04 & 6 & 14 & 32 \\
\hline \multirow[t]{2}{*}{ Occipital } & L Middle Occipital Gyrus & 40 & 3.433 & $5 \mathrm{E}-04$ & -22 & -96 & 16 \\
\hline & L Cuneus & 19 & 3.350 & 6E-04 & -6 & -84 & 12 \\
\hline Temporal & R Middle Temporal Gyrus & 10 & 3.439 & $4 \mathrm{E}-04$ & 60 & -58 & 8 \\
\hline
\end{tabular}

k, cluster size; peak T, t-value at the peak voxel; peak p, p-value at the peak voxel; $x, y, z$, MNI coordinates of the peak voxel.

characteristics of spaceflight such as foot sole unloading, fluid shifts, and sensorimotor adaptation, but not all the features of spaceflight. Second, as revealed by EEG studies, microgravity effects on brain activation are task dependent (Cheron et al., 2006, 2014). Thus the factors influencing neural activation during spaceflight could differ from those reported here. Even in the microgravity environment itself behavior can be context specific. For example, during spaceflight, up-down turning asymmetry was immediately reduced when astronauts were free-floating as compared to tethered (De Saedeleer et al., 2013).The current study is also limited by the inequality in the number of sessions for the HDBR and control groups. Future studies using equal numbers of sessions and similar timelines for both groups would provide more precise information about the temporal dynamics of HDBR effects on dual-tasking behavior. Furthermore, in the current study, only male subjects were included in the HDBR group, as they were serving as controls for a separate investigator's study on testosterone to reduce HDBR decrements. Future studies involving both males and females would provide more comprehensive information and could better generalize to the broader astronaut population.

\section{CONCLUSION}

The current study investigated the effect of 70-day HDBR on dual task performance and the underlying brain activation. 
TABLE 9 | Regions showing positive correlations between pre-to-post HDBR changes in dual-task cost of tapping reaction time and dual-task cost of brain activation.

\begin{tabular}{|c|c|c|c|c|c|c|c|}
\hline Region & & $k$ & Peak $T$ & Peak $p$ & & $\mathrm{z}, \mathrm{z}(\mathrm{mn}$ & \\
\hline \multirow[t]{4}{*}{ Frontal } & R Superior Frontal Gyrus & 113 & 5.383 & 8E-05 & 2 & 28 & 50 \\
\hline & R Superior Frontal Gyrus & 28 & 5.038 & $1 E-04$ & 12 & 14 & 58 \\
\hline & R Inferior Frontal Gyrus & 40 & 5.102 & $1 E-04$ & 44 & 10 & 16 \\
\hline & L Operculum & 12 & 4.935 & 2E-04 & -36 & -8 & 22 \\
\hline \multirow[t]{5}{*}{ Parietal } & L Operculum & 149 & 9.806 & 2E-07 & -44 & -18 & 26 \\
\hline & R Postcentral Gyrus & 129 & 5.811 & 4E-05 & 34 & -20 & 36 \\
\hline & R Inferior Parietal Lobule & 24 & 5.059 & $1 E-04$ & 36 & -36 & 42 \\
\hline & R Precuneus & 10 & 4.308 & 5E-04 & 20 & -46 & 36 \\
\hline & L Angular Gyrus & 32 & 4.732 & 2E-04 & -34 & -52 & 26 \\
\hline \multirow[t]{4}{*}{ Cingulate } & L Middle Cingulate Gyrus & 297 & 7.641 & 3E-06 & -18 & -14 & 46 \\
\hline & L Anterior Cingulate Gyrus & 51 & 5.097 & $1 \mathrm{E}-04$ & -6 & 16 & 26 \\
\hline & R Middle Cingulate Gyrus & 26 & 4.208 & 6E-04 & 18 & 10 & 36 \\
\hline & R Middle Cingulate Gyrus & 42 & 10.446 & 1E-07 & 16 & -38 & 40 \\
\hline \multirow[t]{3}{*}{ Occipital } & R Lingual Gyrus & 11 & 4.237 & 6E-04 & 30 & -63 & 6 \\
\hline & R Cuneus & 14 & 4.268 & 5E-04 & 22 & -72 & 26 \\
\hline & R Lingual Gyrus & 43 & 4.817 & 2E-04 & 28 & -51 & -1 \\
\hline \multirow[t]{3}{*}{ Temporal } & R Middle Temporal Gyrus & 512 & 6.308 & 2E-05 & 41 & -45 & 0 \\
\hline & R Middle Temporal Gyrus & 228 & 5.595 & 6E-05 & 50 & -63 & 0 \\
\hline & L Middle Temporal Gyrus & 476 & 5.757 & $5 E-05$ & -51 & -70 & 3 \\
\hline Insula & L Insula & 26 & 4.462 & 4E-04 & -30 & 16 & 10 \\
\hline \multirow[t]{2}{*}{ Cerebellum } & R Cerebellum Crus I & 60 & 5.275 & $1 E-04$ & 44 & -60 & -29 \\
\hline & L Cerebellum IX & 14 & 4.119 & 7E-04 & -11 & -54 & -42 \\
\hline
\end{tabular}

k, cluster size; peak T, t-value at the peak voxel; peak p, p-value at the peak voxel; $x, y, z$, MNI coordinates of the peak voxel.

Impairments of dual task performance were found in HDBR subjects compared to control subjects. Furthermore, we observed increased activations for dual tasking during HDBR in a widely distributed network involving frontal, parietal, occipital, temporal, cingulate, cerebellar, and subcortical regions. By comparing with activation change in control subjects, we demonstrated that the HDBR-associated brain activation increase was due to the HDBR intervention itself, rather than repeated testing. These results demonstrate lower neural efficiency for dual task processing during HDBR. As a microgravity analog, HDBR has successfully simulated a variety of microgravity exposure effects on human physiology and cognition. Therefore, we conjecture similar behavioral and neural changes occur during spaceflight to those during HDBR. This hypothesis warrants further investigation based on data from astronauts before, during and after their space missions.

\section{REFERENCES}

Adcock, R. A., Constable, R. T., Gore, J. C., and Goldman-Rakic, P. S. (2000). Functional neuroanatomy of executive processes involved in

\section{AUTHOR CONTRIBUTIONS}

PY analyzed data and wrote the manuscript, VK analyzed data and wrote the manuscript, PR designed the study and wrote the manuscript, YD collected data and wrote the manuscript, NG collected data and wrote the manuscript, SW, RR, IK, JB, AM, and RS designed the study and wrote the manuscript.

\section{FUNDING}

This work is supported by grants from the National Space Biomedical Research Institute (NASA NCC 9-58, MA02701, and PF04101), from the National Aeronautics and Space Administration (NASA; NNX11AR02G) and NASA Flight Analogs Project, and the National Institutes of Health, and National Center for Advancing Translational Sciences, 1UL1RR029876-01. dual-task performance. Proc. Natl. Acad. Sci. U.S.A. 97, 3567-3572. doi: $10.1073 /$ pnas.060588897

Al-Yahya, E., Dawes, H., Smith, L., Dennis, A., Howells, K., and Cockburn, J. (2011). Cognitive motor interference while walking: a systematic 
review and meta-analysis. Neurosci. Biobehav. Rev. 35, 715-728. doi: 10.1016/j.neubiorev.2010.08.008

At'kov, O. I., and Bednenko, V. S. (1992). Hypokinesia and Weightlessness: Clinical and Physiologic Aspects. Madison, WI: International Universities Press.

Avants, B. B., Tustison, N. J., Song, G., Cook, P. A., Klein, A., and Gee, J. C. (2011). A reproducible evaluation of ANTs similarity metric performance in brain image registration. Neuroimage 54, 2033-2044. doi: 10.1016/j.neuroimage.2010.09.025

Basner, M., Dinges, D. F., Mollicone, D., Ecker, A., Jones, C. W., Hyder, E. C., et al. (2013). Mars 520-d mission simulation reveals protracted crew hypokinesis and alterations of sleep duration and timing. Proc. Natl. Acad. Sci. U.S.A. 110, 2635-2640. doi: 10.1073/pnas.1212646110

Bock, O., Weigelt, C., and Bloomberg, J. J. (2010). Cognitive demand of human sensorimotor performance during an extended space mission: a dual-task study. Aviat. Space Environ. Med. 81, 819-824. doi: 10.3357/ASEM.2608.2010

Buckey, J. C. (2006). Space Physiology. Cary, NC: Oxford University Press.

Caprihan, A., Sanders, J. A., Cheng, H. A., and Loeppky, J. A. (1999). Effect of head-down tilt on brain water distribution. Eur. J. Appl. Physiol. Occup. Physiol. 79, 367-373. doi: 10.1007/s004210050522

Chen, S., Zhou, R., Xiu, L., Chen, S., Chen, X., and Tan, C. (2013). Effects of 45-day$6^{\circ}$ head-down bed rest on the time-based prospective memory. Acta Astronaut. 84, 81-87. doi: 10.1016/j.actaastro.2012.10.040

Cheron, G., Leroy, A., De Saedeleer, C., Bengoetxea, A., Lipshits, M., Cebolla, A., et al. (2006). Effect of gravity on human spontaneous $10-\mathrm{Hz}$ electroencephalographic oscillations during the arrest reaction. Brain Res. 1121, 104-116. doi: 10.1016/j.brainres.2006.08.098

Cheron, G., Leroy, A., Palmero-Soler, E., De Saedeleer, C., Bengoetxea, A., Cebolla, A. M., et al. (2014). Gravity influences top-down signals in visual processing. PLoS ONE 9:e82371. doi: 10.1371/journal.pone.0082371

De Saedeleer, C., Vidal, M., Lipshits, M., Bengoetxea, A., Cebolla, A. M., Berthoz, A., et al. (2013). Weightlessness alters up/down asymmetries in the perception of self-motion. Exp. Brain Res. 226, 95-106. doi: 10.1007/s00221-013-3 414-7

D'Esposito, M., Detre, J. A., Alsop, D. C., Shin, R. K., Atlas, S., and Grossman, M. (1995). The neural basis of the central executive system of working memory. Nature 378, 279-281. doi: 10.1038/378279a0

Diedrichsen, J. (2006). A spatially unbiased atlas template of the human cerebellum. Neuroimage 33, 127-138. doi: 10.1016/j.neuroimage.2006. 05.056

Dux, P. E., Tombu, M. N., Harrison, S., Rogers, B. P., Tong, F., and Marois, R. (2009). Training improves multitasking performance by increasing the speed of information processing in human prefrontal cortex. Neuron 63, 127-138. doi: 10.1016/j.neuron.2009.06.005

Erickson, K. I., Colcombe, S. J., Wadhwa, R., Bherer, L., Peterson, M. S., Scalf, P. E., et al. (2007). Training-induced functional activation changes in dual-task processing: an FMRI study. Cereb. Cortex 17, 192-204. doi: 10.1093/cercor/bhj137

Feige, B., Scheffler, K., Esposito, F., Di Salle, F., Hennig, J., and Seifritz, E. (2005). Cortical and subcortical correlates of electroencephalographic alpha rhythm modulation. J. Neurophysiol. 93, 2864-2872. doi: 10.1152/jn.0072 1.2004

Foley, C. M., Mueller, P. J., Hasser, E. M., and Heesch, C. M. (2005). Hindlimb unloading and female gender attenuate baroreflex-mediated sympathoexcitation. Am. J. Physiol. Regul. Integr. Comp. Physiol. 289, R1440-R1447. doi: 10.1152/ajpregu.00356.2005

Fowler, B., Bock, O., and Comfort, D. (2000). Is dual-task performance necessarily impaired in space? Hum. Factors 42, 318-326. doi: $10.1518 / 001872000779656507$

Friston, K., Ashburner, J., Frith, C. D., Poline, J. B., Heather, J. D., and Frackowiak, R. S. (1995). Spatial registration and normalization of images. Hum. Brain Mapp. 3, 165-189.

Koppelmans, V., Erdeniz, B., De Dios, Y. E., Wood, S. J., Reuter-Lorenz, P. A., Kofman, I., et al. (2013). Study protocol to examine the effects of spaceflight and a spaceflight analog on neurocognitive performance: extent, longevity, and neural bases. BMC Neurol. 13:205. doi: 10.1186/1471-2377-13-205

Koppelmans, V., Mulavara, A. P., Yuan, P., Cassady, K. E., Cooke, K. A., Wood, S. J., et al. (2015). Exercise as potential countermeasure for the effects of 70 days of bed rest on cognitive and sensorimotor performance. Front. Syst. Neurosci. 9:121. doi: 10.3389/fnsys.2015.00121

Lugg, D. J. (2005). Behavioral health in Antarctica: implications for long-duration space missions. Aviat. Space Environ. Med. 76, B74-B77.

Manzey, D., and Lorenz, B. (1998). Mental performance during short-term and long-term spaceflight. Brain Res. Rev. 28, 215-221. doi: 10.1016/S01650173(98)00041-1

Manzey, D., Lorenz, B., and Poljakov, V. (1998). Mental performance in extreme environments: results from a performance monitoring study during a 438-day spaceflight. Ergonomics 41, 537-559. doi: 10.1080/001401398186991

Manzey, D., Lorenz, B., Schiewe, A., Finell, G., and Thiele, G. (1995). Dual-task performance in space: results from a single-case study during a short-term space mission. Hum. Factors 37, 667-681.

Marois, R., Larson, J. M., Chun, M. M., and Shima, D. (2006). Response-specific sources of dual-task interference in human pre-motor cortex. Psychol. Res. 70, 436-447. doi: 10.1007/s00426-005-0022-6

Mulavara, A. P., Feiveson, A. H., Fiedler, J., Cohen, H., Peters, B. T., Miller, C., et al. (2010). Locomotor function after long-duration space flight: effects and motor learning during recovery. Exp. Brain Res. 202, 649-659. doi: 10.1007/s00221010-2171-0

Navasiolava, N. M., Custaud, M. A., Tomilovskaya, E. S., Larina, I. M., Mano, T., Gauquelin-Koch, G., et al. (2011). Long-term dry immersion: review and prospects. Eur. J. Appl. Physiol. 111, 1235-1260. doi: 10.1007/s00421-01 $0-1750-x$

Nicogossian, A. E., Huntoon, C. L., and Pool, S. L. (1994). Space Physiology and Medicine. Philadelphia, PA: Lea and Fibiger.

Pavy-Le Traon, A., Heer, M., Narici, M. V., Rittweger, J., and Vernikos, J. (2007). From space to Earth: advances in human physiology from 20 years of bed rest studies (1986-2006). Eur. J. Appl. Physiol. 101, 143-194. doi: 10.1007/s00421007-0474-z

Ploutz-Snyder, L. L., Downs, M., Ryder, J., Hackney, K., Scott, J., Buxton, R., et al. (2014). Integrated resistance and aerobic exercise protects fitness during bed rest. Med. Sci. Sports Exerc. 46, 358-368. doi: 10.1249/MSS.0b013e3182a $62 \mathrm{f} 85$

Rao, L. L., Zhou, Y., Liang, Z. Y., Rao, H., Zheng, R., Sun, Y., et al. (2014). Decreasing ventromedial prefrontal cortex deactivation in risky decision making after simulated microgravity: effects of -6 degrees head-down tilt bed rest. Front. Behav. Neurosci. 8:187. doi: 10.3389/fnbeh.2014.00187

Riby, L. M., Perfect, T. J., and Stollery, B. T. (2004). The effects of age and task domain on dual task performance: a meta-analysis. Eur. J. Cogn. Psychol. 16, 863-891. doi: 10.1080/09541440340000402

Roberts, D. R., Ramsey, D., Johnson, K., Kola, J., Ricci, R., Hicks, C., et al. (2010). Cerebral cortex plasticity after 90 days of bed rest: data from TMS and fMRI. Aviat. Space Environ. Med. 81, 30-40. doi: 10.3357/ASEM.253 2.2009

Seidler, R., Purushotham, A., Kim, S.-G., Uğurbil, K., Willingham, D., and Ashe, J. (2002). Cerebellum activation associated with performance change but not motor learning. Science 296, 2043-2046. doi: 10.1126/science.1068524

Shehab, R. L., Schlegel, R. E., Schiflett, S. G., and Eddy, D. R. (1998). The NASA performance assessment workstation: cognitive performance during head-down bed rest. Acta Astronaut. 43, 223-233.

Strangman, G. E., Sipes, W., and Beven, G. (2014). Human cognitive performance in spaceflight and analogue environments. Aviat. Space Environ. Med. 85, 1033-1048. doi: 10.3357/ASEM.3961.2014

Strayer, D. L., Drews, F. A., and Johnston, W. A. (2003). Cell phone-induced failures of visual attention during simulated driving. J. Exp. Psychol. Appl. 9, 23-32. doi: 10.1037/1076-898x.9.1.23

Szameitat, A. J., Schubert, T., Muller, K., and Von Cramon, D. Y. (2002). Localization of executive functions in dual-task performance with fMRI. J. Cogn. Neurosci. 14, 1184-1199. doi: 10.1162/089892902760 807195

Tombu, M., and Jolicoeur, P. (2003). A central capacity sharing model of dualtask performance. J. Exp. Psychol. Hum. Percept. Perform. 29, 3-18. doi: 10.1037/0096-1523.29.1.3

Tustison, N. J., Avants, B. B., Cook, P. A., Zheng, Y., Egan, A., Yushkevich, P. A., et al. (2010). N4ITK: improved N3 bias correction. IEEE Trans. Med. Imaging 29(6), 1310-1320. doi: 10.1109/TMI.2010.2046908 
Verhaeghen, P., Steitz, D. W., Sliwinski, M. J., and Cerella, J. (2003). Aging and dual-task performance: a meta-analysis. Psychol. Aging 18:443. doi: 10.1037/0882-7974.18.3.443

Wenzel, J., Luks, N., Plath, G., Wilke, D., and Gerzer, R. (1998). The influence of $\mathrm{CO} 2$ in a space-like environment: study design. Aviat. Space Environ. Med. 69, 285-290.

Wilkerson, M. K., Muller-Delp, J., Colleran, P. N., and Delp, M. D. (1999). Effects of hindlimb unloading on rat cerebral, splenic, and mesenteric resistance artery morphology. J. Appl. Physiol. 87, 2115-2121.

Wu, T., Liu, J., Hallett, M., Zheng, Z., and Chan, P. (2013). Cerebellum and integration of neural networks in dual-task processing. Neuroimage 65, 466-475. doi: 10.1016/j.neuroimage.2012.10.004
Conflict of Interest Statement: The authors declare that the research was conducted in the absence of any commercial or financial relationships that could be construed as a potential conflict of interest.

Copyright (c) 2016 Yuan, Koppelmans, Reuter-Lorenz, De Dios, Gadd, Wood, Riascos, Kofman, Bloomberg, Mulavara and Seidler. This is an open-access article distributed under the terms of the Creative Commons Attribution License (CC $B Y)$. The use, distribution or reproduction in other forums is permitted, provided the original author(s) or licensor are credited and that the original publication in this journal is cited, in accordance with accepted academic practice. No use, distribution or reproduction is permitted which does not comply with these terms. 